\title{
Una estrategia de diseminación en la psicología: los talleres interactivos
}

Kalina Isela Martínez-Martínez, Lic en Psic, ${ }^{(1)}$ C ésar Carrascosa-Venegas, Mtro en Psic, ${ }^{(1)}$ Héctor Ayala-Velázquez, Dr en Psic..(1) $†$

Martínez-Martínez KI, Carrascosa-Venegas C, Ayala-Velázquez $\mathrm{H}$. Una estrategia de diseminación en la psicología: los talleres interactivos. Salud Publica Mex 2003;45:5-12.

El texto completo en inglés de este artículo está disponible en: http://www.insp.mx/salud/index.html

\section{Resumen}

Objetivo. Evaluar los talleres interactivos como una estrategia de diseminación de un modelo de intervención psicológica para el tratamiento de los bebedores problema, en profesionales de la salud del Instituto Mexicano del Seguro So cial. Material y métodos. Se trabajó con 206 profesionales de la salud de siete clínicas del Instituto Mexicano del Seguro So cial, en el perio do 1999-2000. Los sujetos fueron seleccionados por las autoridades de las clínicas. El tipo de estudio utilizado fue cuasi-experimental con un diseño pretest/pos-test. Inicialmente se aplicó a los participantes el cuestionario de actitudes, intereses y conocimientos, luego se impartieron los talleres interactivos, y al final de éstos nuevamente se aplicó el cuestionario para identificar cambios. El análisis estadístico se realizó a través de una prueba t de student de muestras pareadas. Resultados. Se encontraron diferencias estadísticas significativas en los conocimientos de los participantes sobre alcoholismo $t(206,205)$, $y$ en los intereses de los mismost $(206,205)=2.318, p=0.021$. Conclusiones. El taller interactivo es una herramienta efectiva para diseminar el programa de Auto-C ambio Dirigido en los escenarios de las clínicas del Instituto Mexicano del Seguro Social, por lo que los profesionales de la salud pueden convertirse en agentes de cambio de las innovaciones psicológicas. El texto completo en inglés de este artículo está disponible en: http://www.insp.mx/salud/index.html

Palabras clave: diseminación de información; taller inter activo; bebedor problema; personal de salud; México

\author{
Martínez-Martínez KI, Carrascosa-Venegas C, \\ Ayala-Velázquez $\mathrm{H}$. \\ Interactive workshops as a dissemination \\ strategy in psychology. \\ Salud Publica Mex 2003;45:5-12. \\ The English version of this paper \\ is available at: http://www.insp.mx/salud/index.html
}

\begin{abstract}
A bstract
Objective. To assess whether interactive workshops are an effective strategy for promoting a psychological intervention model among healthcare providers, to treat problem drinkers. Material and Methods The study was conducted between the years 1999 and 2000, among 206 healthcare providers at seven Instituto Mexicano del Seguro Social (Mexican Institute of Social Security, IMSS) clinics. Study subjects were selected by hospital executive officers. The study design is a quasi-experimental pre-test/post-test study. Data on providers' attitudes, interests, and knowledge were collected using a questionnaire.After that, interactive workshops were conducted, and the same questionnaire was applied again at the end of the workshops. Statistical analysis was carried out using Student's t test for matched samples. Results Statistically significant differences were found in participants' knowledge on alcoholism t $(206,205)=-9.234, p=0.001$, as well as in their interest $\mathrm{t}(206,205)=-2.318, p=0.021$. Conclusions. Interactive workshops are an effective tool to disseminate the $G$ uided Self-H elp Program conducted in IMSS clinics. Healthcare providers can become change-inducing/pro moting agents of psychological innovations. The English version of this paper is available at: http://www.insp.mx/salud/index.html
\end{abstract}

Key words: dissemination; interactive workshops; problem drinkers; health personnel; Mexico

El presente estudio fue posible gracias al apoyo recibido a través del Proyecto G 26306-H otorgado al último autor por el Consejo N acional de Ciencia y Tecnología.

(1) Facultad de Psicología. Universidad N acional Autónoma de México, México, D.F., México.

Fecha de recibido: 7 de enero de 2002 - Fecha de aprobado: 12 de septiembre de 2002

Solicitud de sobretiros: Dr. Héctor Ayala-Velázquez. Facultad de Psicología. Universidad N acional Autónoma de México. Avenida Universidad N 0. 3004, Edificio D, Primer piso, Cubículo 17. Colonia Copilco, 04510. México, D.F., México.

Correo electrónico: ayavel@ servidor.unam.mx 
L a diseminación de los resultados de la experimentación es un componente fundamental de la investigación científica. En la ciencia en general, pero en las intervenciones psicológicas en particular, las condiciones actuales demandan sensibilidad de los investigadores para la diseminación de sus trabajos. Actualmente, es necesario asegurar la aplicación de los resultados en todas las disciplinas científicas, y de las técnicas y tratamientos avalados de manera empírica en la psicología.

\section{El problema de la diseminación en la psicología}

Las investigaciones sobre intervenciones psicológicas han resultado en grandes avances en el tratamiento de una amplia gama de desórdenes psicológicos. Estos logros son posibles, sobre todo, en la medida en que sus técnicas de intervención se encuentran sólidamente sustentadas en la investigación empírica. Sin embargo, la utilización de estas técnicas no ha sido la esperada, fundamentalmente por una deficiente diseminación de los hallazgos y técnicas de la investigación clínica.

Un problema relativamente común en el campo de la psicología consiste en que los profesionales no emplean los hallazgos de la investigación clínica; su práctica profesional se caracteriza por un eclecticismo fundado sobre todo en el "ensayo y error". Barlow ${ }^{1}$ señala que esta situación debe resultar particularmente perturbadora "para una disciplina cuya meta ha sido formar profesionales que pudieran integrar los métodos de la ciencia con la práctica clínica para producir nuevos conocimientos".

\section{Importancia de la investigación en diseminación}

La diseminación de los hallazgos y técnicas de la investigación psicológica no siempre recibe la atención necesaria, sobre todo en términos de la identificación y promoción de los medios efectivos para llevarla a cabo, meta que sólo puede alcanzarse mediante la investigación empírica. $\mathrm{McCrady}{ }^{2}$ percibe el problema de la diseminación de la siguiente manera: "nuestro trabajo de investigación es impecable; nuestras aproximaciones a los tratamientos son creativas y efectivas. Nuestra teoría es cuidadosa y meticulosa. Desafortunadamente, nuestro trabajo también es invisible". Por ello se vuelve necesario encontrar formas efectivas de diseminar, en general para los practicantes del campo ${ }^{3}$ los procedimientos para el cuidado de la salud.
Persons ${ }^{4}$ señala que muchas intervenciones efectivas desarrolladas por investigadores en psicología, rara vez se usan en la práctica clínica. Por ejemplo, en el tratamiento del abuso del alcohol, ${ }^{5}$ en el área del tratamiento de las fobias y el pánico, ${ }^{6}$ en el tratamiento de pacientes con padecimientos psiquiátricos, ${ }^{7}$ y en el tratamiento de problemas infantiles. ${ }^{8}$

El desconocimiento de las técnicas y tratamientos empíricamente obtenidos señala la existencia de una brecha entre los campos experimental y aplicado. Por lo tanto resulta esencial cerrar la brecha, es decir, incluir a los profesionales de la salud como parte complementaria del proceso de investigación, ${ }^{9}$ y con ello, cubrir el objetivo de la diseminación y aplicación de técnicas y tratamientos efectivos.

\section{Naturaleza de la brecha entre investigadores y profesionales de la salud}

Crosswaitie y Curtice $^{10}$ afirman que los resultados de la investigación en el campo de la promoción de la salud requieren mayor claridad por parte de los investigadores. Los autores identificaron tres estrategias: 1) definir la diseminación e identificar las barreras a la diseminación efectiva (tomando como punto de referencia la obra de Rogers); ${ }^{11}$ 2) describir las audiencias pertinentes a la investigación en promoción de la salud e identificar sus necesidades, y 3 ) identificar las estrategias adecuadas para implantar la diseminación y promover la utilización de los resultados de la investigación.

Particularmente, en el contexto de las conductas adictivas, y en específico en el consumo excesivo de alcohol, resulta necesario que se incorporen tratamientos desarrollados con bases empíricas en programas de atención, y que se evalúe su efectividad y duración en escenarios reales.

La necesidad de desarrollar estrategias efectivas y eficientes para la diseminación de innovaciones acerca del cuidado de la salud en general, basada en evidencia empírica, ha llevado a explorar tanto las dificultades metodológicas como sociopolíticas que impiden la diseminación y la evaluación de las mismas, ${ }^{12}$ así como los obstáculos a las innovaciones. ${ }^{13}$ La búsqueda de estas estrategias ha llevado incluso a proponer la necesidad de aprender "una nueva manera de hacer negocios". ${ }^{14} \mathrm{El}$ "mercadeo" del investigador conductual ha sido deficiente ya que "no hemos estado cerca de nuestros consumidores". Producir tratamientos fundados empíricamente es sólo una parte de la investigación aplicada en psicología. La otra consiste en que los métodos cumplan con las expectativas espe- 
radas del cuidado de la salud. Esto puede lograrse adoptando como un valor la importancia de diseminar las intervenciones psicológicas en la práctica clíni$\mathrm{ca}$, entrenando a los graduados en nuevas formas de desarrollar su ciencia, en nuevas formas de presentarla a la comunidad y en cómo beneficiarse recíprocamente de la asociación con los profesionales de la salud. ${ }^{15}$

\section{Importancia de los talleres interactivos como recurso para diseminar}

Para resolver este problema, la Division of Clinical Psychology de la American Psychological Association ${ }^{16}$ recomienda formas efectivas de llevar a cabo la diseminación, una vez que se han identificado los tratamientos efectivos para desórdenes específicos, basados en la evidencia actual.

La División of Clinical Psychology desarrolló un programa con varias recomendaciones: la promoción del desarrollo de la competencia de los profesionales de la salud para practicar nuevos procedimientos a través de los programas de educación continua (que combinen aprendizaje didáctico estructurado con trabajo clínico supervisado), y actividades que pueden realizarse sin costo elevado y en poco tiempo con audiencias numerosas, como son los talleres, y la capacitación en la aplicación de estas estrategias, seguidos por la supervisión de los resultados de estas aplicaciones.

En la literatura del área se mencionan diversos antecedentes de la realización de talleres con propósitos de diseminación, por ejemplo, Rivers, Sarata y Book ${ }^{17}$ examinaron los efectos de un taller acerca del alcoholismo en relación con las actitudes, satisfacción y niveles de ejecución de personal secretarial en instituciones que trataban con problemas relacionados con el consumo de alcohol. Los resultados mostraron que el taller tuvo un efecto positivo al incrementar el conocimiento de las secretarias respecto de los problemas del consumo de alcohol.

Crosswaitie y Curtice ${ }^{10}$ reconocieron los problemas prácticos existentes en la implantación de las estrategias de diseminación, asumiendo como premisa básica de su proyecto que la investigación en servicios de salud fuera promovida en términos de la utilidad de su proceso y sus hallazgos hacia los diseñadores de políticas, funcionarios y profesionales de la salud. Parte fundamental del proyecto de diseminación fue el desarrollo de un taller que proporcionó la oportunidad de interactuar y debatir tanto a investigadores como a los profesionales de la salud.

Por último, Shanley, Lodge y Mattick, ${ }^{18}$ emplearon un taller informativo e interactivo como metodología básica de su proyecto, siguiendo la sugerencia de Rogers ${ }^{11}$ acerca de que el mayor impacto sobre los usuarios potenciales de la investigación ocurre cuando los investigadores interactúan de manera sostenida con los primeros.

En conjunto, los resultados de estas investigaciones reportan la efectividad de estrategias de diseminación, como el uso de talleres interactivos, en cumplir con el objetivo de informar y promover procedimientos psicológicos validados empíricamente.

Sin embargo, y a pesar de esta evidencia de efectividad, en México la investigación en diseminación, al igual que en muchos países, es virtualmente nula. Los esfuerzos de diseminación en las diversas disciplinas han transitado por los cursos tradicionales y su impacto en la sociedad ha sido pobre en el cuidado de la salud en general. Estos esfuerzos por diseminar no se han sistematizado adecuadamente. Es decir, las técnicas o procedimientos de intervención no siempre se han derivado de manera experimental; tampoco se ha validado de manera rigurosa su eficacia.

Uno de los trabajos de diseminación en nuestro país es el "Proyecto colaborativo entre el Instituto Mexicano del Seguro Social (IMSS) y la Universidad Nacional Autónoma de México (UNAM): "Desarrollo, Evaluación y Diseminación de un Modelo de Prevención Secundaria para Bebedores Problema en el IMSS", auspiciado por el Consejo Nacional de Ciencia y Tecnología (CONACyT). El objetivo principal del proyecto consistió en desarrollar, evaluar y diseminar un programa de prevención secundaria de corte breve basado en el modelo de Auto-Cambio Dirigido a Bebedores Problema ${ }^{19}$ en clínicas de atención primaria a la salud, del Instituto Mexicano del Seguro Social, en la zona metropolitana de la Ciudad de México y en otros centros urbanos del interior de la República.

Entre las diversas estrategias de diseminación aplicadas se eligió la realización de talleres interactivos, en función de los promisorios resultados obtenidos, citados anteriormente, y en virtud de la relación estrecha que habría de establecerse entre los actores directamente involucrados en el proceso (profesionales de la salud). El propósito de este trabajo fue evaluar los talleres interactivos como estrategia de diseminación del "modelo Auto-Cambio Dirigido para Bebedores Problema" en profesionales de la salud del IMSS.

\section{Material y métodos}

\section{Sujetos}

En el periodo 1999-2000 participaron 206 sujetos seleccionados por las autoridades de las clínicas del IMSS, de acuerdo con el interés reportado por el personal de 
salud en conocer y participar en la capacitación sobre un modelo de prevención secundaria para bebedores problema. La convocatoria se realizó por invitación abierta, sin límite de participantes, con la intención de hacerlo llegar a la mayor cantidad posible de profesionales de la salud.

\section{Escenario}

Los talleres interactivos se llevaron a cabo en diversas clínicas del primer nivel de atención del IMSS, dentro de las salas de juntas, asignadas por los directivos. Los estados donde se aplicó el taller interactivo fueron el Distrito Federal, Hidalgo, Querétaro, Morelos y Tlaxcala.

\section{Instrumentos}

I. Cuestionario de actitudes, intereses y conocimientos. Para la evaluación del impacto del taller se elaboró un instrumento con tres áreas: actitudes, conocimientos e intereses. La consistencia interna del instrumento fue de alfa 0.70 .

a) Area de actitudes (15 reactivos). Esta evalúa las actitudes del profesional de la salud en tres dimensiones:

i) Evaluación del bebedor problema y sus características (tres reactivos).

ii) Papel del profesional de la salud en el tratamiento de los bebedores problema (siete reactivos).

iii) Evaluación de la evidencia empírica de la efectividad de diferentes tratamientos para el consumo problemático de alcohol (cinco reactivos). Sin embargo, no se reportan resultados del área de actitudes puesto que no es el objetivo.

b) Area de conocimientos (ocho reactivos). Se elaboraron preguntas para evaluar la información respecto al programa de Auto-Cambio Dirigido para Bebedores Problema. El instrumento evalúa los conocimientos acerca del programa en tres dimensiones:

i) Meta del tratamiento (moderación o abstinencia).

ii) El papel de las recaídas en el proceso de tratamiento.

iii) Importancia de la evaluación del patrón de consumo (cantidad, frecuencia y situaciones de consumo). c) Area de interés. Está compuesta por nueve reactivos para identificar el interés del profesional de la salud en las siguientes dimensiones: i) interés por conocer los tratamientos exitosos en relación con los problemas derivados del abuso del alcohol, ii) interés por capacitarse en el programa Auto-Cambio Dirigido para Bebedores Problema, iii) interés por identificar, canalizar y tratar esta problemática dentro del modelo propuesto (Auto-Cambio Dirigido para Bebedores Problema).

II. Instrumento de satisfacción (nueve reactivos). Se elaboró este instrumento para evaluar la satisfacción de los participantes del taller interactivo, en cuanto a las características generales del taller (duración y temas presentados) y técnicas didácticas (lectura, juego de roles, lluvia de ideas). Se evaluó a través de una escala likert que va de "muy satisfactorio" a "nada satisfactorio".

\section{Tipo de estudio}

El tipo de estudio fue cuasi-experimental con un diseno pre-test/pos-test.

\section{Procedimiento}

De acuerdo con el modelo de Rogers, ${ }^{11}$ en el cual se plantea que la diseminación se refiere a la transferencia de la innovación del sistema de recursos al sistema de usuarios, un primer paso de esta investigación fue la realización de los talleres interactivos en clínicas de salud. Los talleres interactivos tuvieron una duración de cinco horas aproximadamente y se llevaron a cabo en una sola sesión de trabajo. Los talleres constaron de tres partes. Durante la primera se efectuaron las siguientes actividades: a) además de las presentaciones de rigor, se llevó a cabo la primera aplicación del instrumento de actitudes, intereses y conocimientos; $b$ ) a continuación se discutió el problema del consumo excesivo de alcohol en México, así como el papel a desempeñar por el profesional de la salud, además de las políticas de salud con respecto a la prevención y tratamiento del consumo excesivo de alcohol.

En la segunda parte se analizaron y discutieron conceptos contrastantes como "alcohólico" versus "bebedor problema" $\mathrm{y}$ "enfermedad progresiva y mortal" versus "conducta aprendida y modificable". Se apoyó esta discusión mediante viñetas que ilustraban casos. Además se presentaron los fundamentos princi- 
pales del modelo de Auto-Cambio Dirigido para Bebedores Problema.

La tercera parte comprendió un "juego de roles" entre uno de los participantes y uno de los facilitadores, con la intención de ilustrar la adecuada identificación y canalización de un bebedor problema. Al finalizar el taller se procedió a la segunda aplicación del cuestionario de actitudes, intereses y conocimientos. Además, se incluyó la evaluación sobre la satisfacción de los participantes con la coordinación del taller interactivo y sus técnicas didácticas. El área de conocimientos del instrumento se puntuó de la siguiente manera: cada reactivo tenía cinco opciones de respuesta que iban de Totalmente en desacuerdo a Totalmente de acuerdo; los reactivos 2, 5 y 6 se calificaron con un punto si el profesional de la salud contestó De acuerdo, y con dos puntos si contestó Totalmente de acuerdo. Los reactivos $1,3,4,7$ y 8 calificaron con un punto si la respuesta fue En desacuerdo, y dos puntos si la respuesta fue Totalmente en desacuerdo. En el área de intereses se le dio un punto a los reactivos si se contestó De acuerdo, y dos puntos si la respuesta fue Totalmente de acuerdo.

\section{Resultados}

Del total de los participantes en los talleres interactivos, 30\% fueron hombres y $70 \%$ mujeres. La edad promedio fue de 38 años, en un rango de 23 a 69 años. La especialidad de los participantes fue de enfermería, $13.1 \%$; trabajo social, $12.6 \%$; medicina familiar, $52.8 \%$; asistencia médica, $8.8 \%$; salud pública, $6.8 \%$, y psicología, $5.9 \%$.

Los resultados del taller interactivo se presentan en el siguiente orden: primero se describen los resul- tados del área de conocimientos e intereses, en los cuales se realizó un análisis estadístico de pruebas $t$ de muestras pareadas antes y después del taller interactivo. Como segundo punto, se reportan los resultados del cuestionario de satisfacción de los participantes con el taller interactivo.

En el área de conocimientos el resultado obtenido de la prueba $t$ de muestras pareadas entre las aplicaciones, antes y al terminar el taller interactivo, mostró una diferencia estadística significativa $t(206,205)=$ -9.234, $p=0.001$; la media de pre fue de 7.3738 y la de post fue de 9.6942. Esto significa un incremento de 2.3204 en el área de conocimientos al final del taller interactivo.

Se realizó un análisis de los reactivos que conforman el área de conocimientos para identificar en cuáles hubo diferencias significativas antes y después del taller interactivo (cuadro I).

En el cuadro I se puede observar que los reactivos $1,2,3,5,6$ y 8 presentaron diferencias significativas antes y después del taller interactivo. Por ejemplo, el reactivo 1 tuvo una media previa al taller de 0.98, y después del taller una media de 1.36, lo que significa que hubo un incremento en el conocimiento de los participantes en el taller en cuanto a las características principales del programa de intervención breve para bebedores problema; en este caso la meta puede ser, además de la abstinencia, la moderación.

\section{Intereses}

Respecto al área que se refiere al interés reportado por el personal de salud para participar en la identificación, canalización y tratamiento de los bebedores problema, el resultado obtenido de la prueba $t$ de

Cuadro I

Comparación de resultados de los reactivos del Área de conocimientos aplicados a personal de SALUd de siete clínicas del Instituto Mexicano del Seguro Social, 1999-2000

Reactivo

\begin{tabular}{|c|c|c|c|c|c|}
\hline \multicolumn{2}{|c|}{ Pre } & \multicolumn{2}{|c|}{ Post } & \multirow[b]{2}{*}{ t } & \multirow[b]{2}{*}{$p$} \\
\hline edia & $\mathrm{DE}$ & Media & $\mathrm{DE}$ & & \\
\hline 0.98 & 0.96 & 1.36 & 0.92 & -4.663 & 0.000 \\
\hline 0.12 & 0.46 & 0.36 & 0.76 & -4.074 & 0.000 \\
\hline 0.21 & 0.59 & 0.66 & 0.93 & -6.231 & 0.000 \\
\hline 1.07 & 0.97 & 1.14 & 0.97 & -0.864 & 0.389 \\
\hline 1.65 & 0.62 & 1.79 & 0.60 & -2.509 & 0.013 \\
\hline 1.01 & 0.86 & 1.65 & 0.71 & -8.510 & 0.000 \\
\hline 1.66 & 0.64 & 1.67 & 0.68 & -0.350 & 0.727 \\
\hline 0.68 & 0.93 & 1.05 & 1.00 & -3.879 & 0.000 \\
\hline
\end{tabular}

$*(<0.05)$ 
muestras pareadas entre las aplicaciones antes y al término del taller interactivo, muestra una diferencia estadística significativa $t(206,205)=-2.318, p=0.021$; la media de pre fue de 16.0243 y la de post fue de 16.5243 .

En el cuadro II se puede observar que los reactivos 3, 5 y 7 presentaron cambios estadísticamente significativos; estos reactivos se refieren a la preocupación que el personal de salud manifiesta por obtener información y enseñar a moderar a sus pacientes con problemas de consumo de alcohol, y la utilización del patrón de consumo para hacer el diagnóstico.

\section{Satisfacción}

Uno de los elementos fundamentales de la aplicación del taller interactivo fue la evaluación de la satisfacción de los participantes al taller en cuanto a las características generales del mismo (duración y temas presentados), y sus técnicas didácticas (lectura, juego de roles, lluvia de ideas). En términos generales, 79\% de los participantes dijeron sentirse satisfechos y muy satisfechos con las características generales del taller y sus técnicas didácticas; $12 \%$ manifestó sentirse medianamente satisfecho y sólo $9 \%$ reportó sentirse poco satisfecho.

\section{Discusión}

Los resultados de la evaluación del taller interactivo se dividen en dos áreas: 1) conocimientos de los profesionales de la salud con relación al modelo de Auto Cambio Dirigido para Bebedores Problema, y 2) el interés reportado por los profesionales de la salud por participar en la identificación, canalización y tratamiento de los bebedores problema a través del modelo de intervención propuesto, y la satisfacción con el taller al que asistieron.

a) El taller interactivo demostró cambios estadísticamente significativos en los "conocimientos" de los participantes acerca de las características de las intervenciones breves para los bebedores problema. Específicamente relacionado a 1) la meta de consumo. Los profesionales de la salud que participaron en el taller interactivo reportaron que dentro del tratamiento de los bebedores problema una alternativa posible es la moderación y no sólo la abstinencia, siendo esto una característica de las intervenciones breves con bebedores problema, ${ }^{20}$ 2) la posibilidad de que el bebedor problema elija su meta de consumo. Esto es, fomentar la

\section{Comparación de resultados de los reactivos del área de interés, aplicados a personal de salud de siete clínicas del Instituto Mexicano del Seguro Social, 1999-2000}

Reactivo

$$
\frac{\text { Pre }}{\text { Media DE }} \frac{\text { Post }}{\text { Media DE }} \quad t \quad p
$$

1. Identificar a las personas que tienen problemas por su consumo de alcohol es útil para brindar un tratamiento adecuado

$\begin{array}{llllll}1.95 & 0.30 & 1.97 & 0.22 & -0.928 & 0.354\end{array}$

2. Enseñar a moderar su consumo de alcohol a personas que tienen problemas por el mismo,

$\begin{array}{lllllll}\text { es importante en mi práctica clínica } & 1.90 & 0.38 & 1.91 & 0.39 & -0.128 & 0.898\end{array}$

3. Me preocupo por obtener información acerca de los diferentes tratamientos

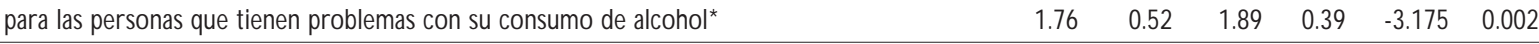

4. Utilizo la información recabada para identificar a los pacientes que tienen problemas con su consumo de alcohol

$\begin{array}{llllll}1.81 & 0.46 & 1.86 & 0.45 & -1.196 & 0.233\end{array}$

5. Utilizo el patrón de consumo de alcohol como una herramienta regular para hacer

$\begin{array}{lllllll}\text { un diagnóstico* } & 1.43 & 0.77 & 1.63 & 0.70 & -3.111 & 0.002\end{array}$

6. Canalizo a mis pacientes que tienen problemas con su consumo de alcohol a asistir

$\begin{array}{lllllll}\text { a tratamiento para cambiar su consumo } & 1.68 & 0.66 & 1.69 & 0.66 & -0.159 & 0.874\end{array}$

7. Me preocupo por enseñar a moderar su consumo de alcohol a mis pacientes que tienen problemas por el mismo*

8. Me interesa colaborar con otros profesionales en el tratamiento de personas que tienen $\begin{array}{lllllll}\text { problemas por su consumo de alcohol } & 1.91 & 0.32 & 1.88 & 0.41 & 0.848 & 0.397\end{array}$

9. Estoy dispuesto a invertir tiempo y esfuerzo en aprender a identificar, canalizar y tratar a personas que tienen problemas con su consumo de alcohol $\quad \begin{array}{lllllll}1.85 & 0.44 & 1.85 & 0.41 & 0.000 & 1.000\end{array}$

$*(>0.05)$ 
responsabilidad de la persona respecto a su conducta de consumo, siendo el individuo un agente activo en el proceso de aprendizaje, y por lo tanto del cambio conductual, las metas representan una norma o modelo interno utilizado por las personas para evaluar su propia ejecución; Miller y Colaboradores ${ }^{5}$ sugieren que cuando los clientes se involucran en el proceso de decisión, éstos se comprometen y realizan mejor su meta que cuando son asignadas por otros. A largo plazo, una meta auto seleccionada fortalece la confianza y ayuda a mantener el cambio de la conducta, y 3) las características del bebedor problema y las diferencias con el concepto de alcoholismo. Es decir, se explicó que los bebedores problema no presentan dependencia severa ni reportan síntomas de retiro; su consumo problemático data de menos de 10 años en promedio y en la mayoría de los casos no han buscado tratamiento previo; usualmente tienen redes sociales y familiares intactas; son pocas las consecuencias que tienen en estos ámbitos por su manera de beber. También es mayor su estabilidad económica y laboral en comparación con quienes tienen una dependencia severa. ${ }^{21}$

b) En cuanto al "interés" reportado por el personal de salud para participar en la identificación, tratamiento y canalización de los bebedores problema, se obtuvieron efectos significativos relacionados con 1) la preocupación que el personal de salud manifiesta por obtener información y enseñar a moderar a sus pacientes con problemas de consumo de alcohol, y 2) la utilización del patrón de consumo para hacer el diagnóstico. En tanto que los reactivos que no mostraron cambios significativos de la primera a la segunda aplicación tienen que ver con el interés reportado por los participantes para capacitarse en el modelo de auto-cambio dirigido. De acuerdo con estos resultados se puede observar que antes del taller interactivo los participantes ya reportaban interés por participar en la capacitación en el programa Auto-Cambio Dirigido para Bebedores Problema. Por lo que este pequeño incremento pudo deberse a que la selección de los miembros se hizo por invitación y no en forma aleatoria, y al asistir al taller interactivo se mantuvo el interés que reportaban antes de participar en éste (ver procedimiento).

El taller proporcionó un escenario interactivo para sensibilizar a los profesionales de la salud en la problemática del consumo de alcohol en México y tener un acercamiento con los investigadores del área, en este caso, el programa Auto-Cambio Dirigido para Bebedores Problema. Además, permitió incluir a los profesionales de la salud como parte complementaria del proceso de investigación, ${ }^{9}$ por la aplicación de técnicas y tratamientos efectivos y su evaluación continua, a través de la supervisión y capacitación en el programa antes mencionado.

c) En general, los participantes en los talleres interactivos reportaron sentirse muy satisfechos con las características generales del taller y sus técnicas didácticas. Es importante resaltar que se impartieron los talleres de acuerdo con lo propuesto por Montgomery y Ayllon, ${ }^{22}$ respecto a la homogeneización del lenguaje y discusión de la relevancia del problema, para lograr una interacción efectiva entre los investigadores del programa para bebedores problema y los profesionales de la salud que laboran en clínicas del Instituto Mexicano del Seguro Social.

De tal forma, los talleres interactivos resultan importantes en la diseminación del programa Auto-Cambio Dirigido para Bebedores Problema puesto que los costos sociales, económicos y de salud asociados con el alcoholismo y abuso del alcohol son incentivos poderosos para el temprano reconocimiento y tratamiento de enfermedades potencialmente curables. Los profesionales de la salud pueden jugar un rol crucial para reducir la morbilidad y mortalidad asociada con el abuso de alcohol; asimismo, tienen la oportunidad especial de preguntar cuestiones personales a sus pacientes y también pueden evaluar aspectos sociales, biológicos o conductuales que indican abuso de alcohol.

\section{Referencias}

1. Barlow DH. On the relation of clinical research to clinical practice: Current issues, new directions. J Consult Clin Psychol 1981;49(2): 147-155.

2. McC rady BS. Implications for behavior therapy of the changing alcoholism health care delivery system. Behav Ther 1986;9:171-179.

3. W ilson KG. The revolution to come: Commentary on "promises to keep". Behav Ther 1997;28:597-600.

4. Persons JB. D issemination of effective methods: Behavior therapy's next challenge. Behav Ther 1997;28:465-471.

5. Miller W R, Brown JM, SimpsonT L, Handmaker N S, BienTH, Luckie LF et al.W hat works? A methodological analysis of the alcohol treatment outcome literature. En: Hester RK, Miller W R, ed. Handbook of alcoholism treatment approaches: Effective alternatives. Boston (MA):Allyn and Bacon, 1995:457-472. 
6. Barlow H. Psychological interventions in the era of managed competition. Clin Psychol Science Pract 1994;1:109-122.

7. Boudewyns SP, FryT, N ightingale E.Token economy programs in theV.A. medical centers:W here are they today? Behav Ther 1986;6:126-127.

8. Rushton HG. N octurnal enuresis: Epidemiology, evaluation, and currently available treatment options. J Pediatr 1989;114:691-696.

9. Barlow DH. Promises to keep. Behav Ther 1997; 28:589-596.

10. Crosswaite $C, C$ urtice L. D isseminating research results - The challenge of bridging the gap between health research and health action. Health Promot Internation 1994;9(4):289-296.

11. Rogers EM. D iffusion of innovations. $4^{\text {th }}$ edition. N uevaYork (N Y): Free Press, 1995.

12. Hayward S, Ciliska D, DiC enso A, Thomas H, Underwood E), Rafael A. Evaluation research in public health: Barriers to the production and dissemination of outcomes data. C an J Public Health 1996;87:413-417.

13. Z ayfert C, Black C. Implementation of empirically supported treatment for PTSD: 0 bstacles and innovations. Behav Ther 2000;23(8): 161-168.

14. Sobell LC. Bridging the gap between scientists and practitioners:The challenge before us. Behav Ther 1996;27:297-320.

15. Goldfried MR, Wolfe BE. Toward a more clinically valid approach to therapy research. J Consult C lin Psychol 1998;66(1):143-150.
16. American Psychological Association. Task force on the promotion and dissemination of psychological procedures. En:The meeting of the American Psychological Association; 1993 A ugust; Toronto, C anadá.

17. Rivers PC, Sarata BP, Book T. Effect of an alcoholism workshop on attitudes, job satisfaction and job performance of secretaries. Q J Stud Alcohol 1974;35:1382-1388.

18.Shanley C, Lodge M, Mattick R. Dissemination of research findings to alcohol and other drug practitioners. DrugAlcohol Rev 1996;15:89-94. 19. Ayala H, Echeverría L, Sobell M, Sobell L. Una alternativa de intervención breve y temprana para bebedores problema en México. Acta Comportamentalia 1998:6:71-93.

20. Ayala H, Echeverría L. D etección temprana e intervención breve. En: Medina-Mora ME, ed. Beber de tierra generosa: ciencia de bebidas alcohólicas en México. México, D.F.: Fundación de Investigaciones Sociales, 1998:148-163.

21. So bell LC, Sobell MB. Guided Self-Change clinical treatment manual. Toronto:Addiction Research Foundation, 1995.

22. Montgomery R, Ayllon T. Matching verbal repertoires: Understanding the contingencies of practice in order to functionally comunicate with clinicians.J Behav Ther Exp Psychiatry 1995;26(2):99-105. 